

\title{
High-speed force spectroscopy unfolds titin at the velocity of molecular dynamics simulations.
}

Felix Rico, Laura Gonzalez, Ignacio Casuso, Manel Puig-Vidal, Simon Scheuring

\section{- To cite this version:}

Felix Rico, Laura Gonzalez, Ignacio Casuso, Manel Puig-Vidal, Simon Scheuring. High-speed force spectroscopy unfolds titin at the velocity of molecular dynamics simulations.: Experiment meets simulation: HS-FS. Science, 2013, 342 (6159), pp.741-3. 10.1126/science.1239764 . inserm-01309044

\section{HAL Id: inserm-01309044 https://www.hal.inserm.fr/inserm-01309044}

Submitted on 28 Apr 2016

HAL is a multi-disciplinary open access archive for the deposit and dissemination of scientific research documents, whether they are published or not. The documents may come from teaching and research institutions in France or abroad, or from public or private research centers.
L'archive ouverte pluridisciplinaire HAL, est destinée au dépôt et à la diffusion de documents scientifiques de niveau recherche, publiés ou non, émanant des établissements d'enseignement et de recherche français ou étrangers, des laboratoires publics ou privés. 


\title{
High-speed force spectroscopy unfolds titin at the velocity of molecular dynamics simulations
}

\author{
Felix Rico ${ }^{1}$, Laura Gonzalez ${ }^{2}$, Ignacio Casuso ${ }^{1}$, Manel Puig-Vidal ${ }^{2}$ \& Simon Scheuring ${ }^{1 *}$ \\ ${ }^{1}$ U1006 INSERM, Université Aix-Marseille, Parc Scientifique et Technologique de Luminy, 163 \\ avenue de Luminy, 13009 Marseille, France \\ ${ }^{2}$ Bioelectronics Group, Department of Electronics, Universitat de Barcelona, c/ Marti Franques \\ 1, 08028 Barcelona, Spain
}

${ }^{*}$ Correspondence should be addressed to S. Scheuring

Email: simon.scheuring@inserm.fr

Tel.: ++33-4-91828777, Fax: ++33-4-91828701 


\section{(Abstract)}

The mechanical unfolding of muscle protein titin by atomic force microscopy (AFM) was a landmark in our developing understanding of single biomolecule mechanics. Molecular dynamics simulations offered atomic-level descriptions of the forced unfolding. However, experiment and simulation could not be directly compared because they differed in pulling velocity by orders of magnitude. We have developed high-speed force spectroscopy (HS-FS) to unfold titin at velocities reached by simulation $(\sim 4 \mathrm{~mm} / \mathrm{s})$. We show that a small $\beta$-strand pair of an Ig domain dynamically unfolds and refolds, buffering pulling forces up to $\sim 100 \mathrm{pN}$. The distance to the unfolding barrier is larger than previously estimated, but in better agreement with atomistic predictions. The ability to directly compare experiment and simulation is likely to be important in studying biomechanical processes.

\section{(OneSentenceSummary)}

Experimentally accessing timescales previously only accessible to simulations suggests roughness in the energy landscape. 


\section{(MainText)}

Titin is a molecular spring in muscle sarcomeres important in striated muscle function and implicated in diseases such as heart failure (1). Titin composes 300 modules including immunoglobulin (Ig)-type, fibronectin III-type and PEVK domains (2). Force spectroscopy (FS) unfolding of individual titin molecules, using optical tweezers $(3,4)$ and AFM $(5)$ opened a new research field relating protein mechanics, structure and folding. AFM force-extension curves revealed saw-tooth-like patterns (periodicity $25-28 \mathrm{~nm}$ ), reporting the unfolding of individual Ig-like domains (5). Combination of AFM experiments with steered molecular dynamics (SMD) simulations enriched atomic level descriptions (6-8) of receptor/ligand binding $(9,10)$ and forced protein unfolding (5). Forced unfolding (pulling speed $0.3-0.5 \mu \mathrm{m} / \mathrm{s}$ ) of titin $191(8,11,12)$ resulted in $\sim 0.7 \mathrm{~nm}$ extension of each domain which correlated with the separation of antiparallel $\beta$ strands $A$ and $B$ observed in SMD simulations $(8,11)$. Subsequent rupture of the A'-G $\beta$-strand pair lead to complete domain unfolding $(11,13,14)$. However, about six orders of magnitude velocity difference prevented direct comparison of SMD with FS. Indeed, simulations resulted in unfolding forces of $\sim 1 \mathrm{nN}$, nearly one order of magnitude higher than experimental values $(11,12)$. Improved computational abilities have allowed simulations which unfolded 191 at $2800 \mu \mathrm{m} / \mathrm{s}$ (still $\sim 2.5$ orders of magnitude faster than experiment) reporting forces of $\sim 500 \mathrm{pN}$ (15).

High-speed AFM (HS-AFM; (16)) allows imaging biomolecules at video rate (1719), through miniaturization of piezoelectric elements and the cantilever (20). Based on HS-AFM, we developed HS-FS with short cantilevers (21). This allowed pulling titin molecules at speeds up to $\sim 4000 \mu \mathrm{m} / \mathrm{s}$, about 2.5 orders of magnitude faster than conventional AFM and reaching current limits for SMD simulations.

Our HS-FS setup is composed of a miniature piezoelectric actuator and a short cantilever with small viscous damping $(0.035 \mathrm{pN} /(\mu \mathrm{m} / \mathrm{s}))$ (Fig. 1A, Fig. S1 and Fig. S2). Using HS-FS titin 191 concatemers were unfolded at pulling velocities 
ranging over six orders of magnitude, from $0.0097 \mu \mathrm{m} / \mathrm{s}$ to $3870 \mu \mathrm{m} / \mathrm{s}$. Only force curves with at least 3 saw-tooth-like unfolding peaks were analyzed (22) (Fig. 1B, Fig. S3). As reported before $(5,23)$, unfolding forces increased with pulling velocity (Fig. 2A). At slow velocities, HS-FS unfolding forces are in excellent agreement with conventional FS (Fig. 2B). At pulling velocities higher than previously (> $100 \mu \mathrm{m} / \mathrm{s}$ ), unfolding forces follow a steeper slope that reach values over $500 \mathrm{pN}$ and overlap with those obtained by simulations (Fig. 2B). A varying slope in the plot of mean rupture forces versus the logarithm of the velocity (force spectrum) have been observed for receptor/ligand interactions (24-26), but have rarely been documented for protein unfolding (27), probably due to the limited range of accessible pulling rates. The microscopic model developed by Hummer and Szabo $(26,28)$ allowed us to fit the wide range of pulling velocities and describes well the nonlinear upturn in the dynamic force spectrum $(26,28)$ (Fig. 2B and SI, Data Analysis). According to this theory, at moderate velocities, unfolding is dominated by the pulling rate and stochastic fluctuations, i.e. spontaneous unfolding of the domain under a given force. At high velocities, stochastic fluctuations of the protein along the unfolding pathway become irrelevant and the unfolding process becomes deterministic (28), because the protein is pulled so fast that it has no time to explore its energy landscape. Importantly, as the slope in the dynamic force spectrum is related to the position of the energy barrier, the slope upturn at high velocities corresponds to a shift of the barrier closer to the native state. From our data, the regime transition occurs at experimental velocities $\sim 1000 \mu \mathrm{m} / \mathrm{s}$ and a critical force of $\sim 350 \mathrm{pN}$ (SI). Our fastest experiment at $3870 \mu \mathrm{m} / \mathrm{s}$ is situated the beginning of the deterministic regime, while most of the HS-FS data points characterize the transition from the stochastic to the deterministic regimes (Fig. 2B). SMD simulations at much higher velocities ( $>1000 \mu \mathrm{m} / \mathrm{s})$ have generally been carried out in this deterministic regime. Although SMD simulation derived forces are in agreement with our fastest pulling data, the theoretically predicted trend deviates from simulations at velocities $>10^{4} \mu \mathrm{m} / \mathrm{s}$. These deviations may be explained by slight differences in the simulated conditions (e.g. temperature) or by the simple cusp-shape of the potential in the 
theory. More refined theories may be necessary to describe the unfolding at very high velocities. The model fit results in an energy landscape where the unfolding transition barrier $\left(x_{\beta}\right)$ is located at $0.89 \mathrm{~nm}$ and the molecular elasticity $\left(k_{m}\right)$ is $376 \mathrm{pN} / \mathrm{nm}$, leading to an unfolding barrier height $(\Delta G)$ of $36 k_{\mathrm{B}} T$, and a spontaneous unfolding rate $k_{0}$ of $2 \cdot 10^{-10} \mathrm{~s}^{-1}$ (Fig. S4). Similar values were obtained by fitting a unified model valid for different potential shapes (28) to the unfolding forces at velocities $\leq 100 \mu \mathrm{m} / \mathrm{s}$, suggesting that the reported parameters are independent of the potential shape (Fig. S6). Our barrier position $(0.89 \mathrm{~nm})$ is larger than previous experimental estimates $(0.25 \mathrm{~nm}(23)$; $0.30 \mathrm{~nm}(5))$ but in better agreement with the distance (1.1-1.4 nm) at which the secondary structure of 191 breaks in simulations $(8,15)$. The relatively narrow range of experimental velocities in former FS experiments did not show an upturn in the force spectra and hence justified the Bell-Evans assumption of a fixed distance to the transition barrier under force. Our experiments at higher velocities show that this assumption is not valid. Furthermore, the data allowed us to estimate a diffusion coefficient of the protein along the reaction coordinate of the free energy landscape $D \sim 4 \times 10^{3} \mathrm{~nm}^{2} / \mathrm{s}(\mathrm{SI})$. This is orders of magnitude slower than diffusion coefficients of proteins in solution $\left(\sim 10^{8} \mathrm{~nm}^{2} / \mathrm{s}\right)(29)$. Slow diffusion has been interpreted as a result of cantilever viscous damping (30) or by local minima along the unfolding pathway (31). Given the much smaller damping coefficient of the cantilevers used here, our data supports the hypothesis that roughness in the free energy landscape slows unfolding. Although our estimated barrier height $\left(36.4 k_{\mathrm{B}} T\right)$ is similar to that measured from chemical unfolding $\left(37 k_{\mathrm{B}} T\right)$, the intrinsic unfolding rate $\left(2 \cdot 10^{-10} \mathrm{~s}^{-1}\right)$ is much slower than estimates from $\mathrm{FS}$ at slow pulling velocity $\left(3.3 \cdot 10^{-4} \mathrm{~s}^{-1}\right)$ and chemical unfolding $\left(4.9 \cdot 10^{-4} \mathrm{~s}^{-1}\right)(23)$. The fast intrinsic dissociation rate from slow FS and Bell-Evans analysis suggests an oversimplified view of forced unfolding, while chemical unfolding explores unrestricted unfolding pathways different from the physiologically relevant directional unfolding during muscle relaxation. Indeed, our slow $k_{0}$ suggests that the titin 191 domains unfold only very rarely at the estimated physiological forces $(\sim 5 \mathrm{pN})$ acting on distal titin lg domains (32). 
The use of short cantilevers with fast response $\left(\tau_{c} \sim 0.7 \mu \mathrm{s}\right.$, Fig. 1) allowed us not only to pull fast but also at conventional velocities (10 to $1000 \mathrm{~nm} / \mathrm{s}$ ) with $\mu \mathrm{s}$ time-resolution. This response time is almost three orders of magnitude shorter than that of conventional cantilevers and allowed estimating a lower limit of the relaxation time of the unfolded polypeptide chain $(<2 \mu \mathrm{s}$, Fig. S3). Before complete domain unfolding, an intermediate state has previously been documented by the so-called "hump" in force curves (Fig. 3A, arrows). This intermediate state is characterized by a force drop in the stretching regime (Fig. 3A, arrows), caused by the separation of the A-B $\beta$-strand pair as revealed by simulations $(11,12)$. HS-FS measurements show separation of the A-B $\beta$ strand pair in several domains within one microsecond (Fig. 3B, first peak). Additionally, at high retraction speeds $(>1 \mathrm{~mm} / \mathrm{s})$, not only the first domain presented a "hump" before unfolding but also consecutive domains. The percentage of domains displaying intermediate unfolding decreased from $\sim 95 \%$ at the lowest to $\sim 40 \%$ at the highest velocities. At $2 \mathrm{~mm} / \mathrm{s}$, the time between domain unfolding is $\sim 10 \mu \mathrm{s}$ (Fig. 3B). Thus, this short time-lapse following the preceding domain unfolding is enough for refolding domains into their native state. This result allows us to set the lower limit for the refolding rate from the intermediate to the native state to at least $\sim 10^{5} \mathrm{~s}^{-1}$, much faster than previous estimates $\left(25 \mathrm{~s}^{-1}\right)(11)$.

We analyzed the intermediate unfolding state up to $2000 \mu \mathrm{m} / \mathrm{s}$, beyond this velocity it is difficult to assess an accurate measurement (Fig. 3, Fig. S3). At conventional pulling velocities the average unfolding forces to the intermediate are independent of pulling rate. At velocities faster than $\sim 100 \mu \mathrm{m} / \mathrm{s}$, average "hump" forces increase drastically, reaching values up to $\sim 300 \mathrm{pN}$ (Fig. 3C), consistent with "hump" forces observed in simulations (15). The slow pulling regime $(<100 \mu \mathrm{m} / \mathrm{s})$ is dominated by near-equilibrium unfolding and refolding of the A-B $\beta$-strand pair and defines the equilibrium force (Fig. 3A). At higher velocities refolding of the $A-B \quad \beta$-strand pair is negligible and the structure unfolds stochastically at forces that increase with the logarithm of the pulling rate (33) (Fig. 3C and SI). The model fitted our results with an unfolding rate at 
zero force from native to intermediate $k_{\mathrm{NI}}{ }^{0}$ of $7 \cdot 10^{3} \mathrm{~s}^{-1}$, an even faster folding rate of $k_{\mathrm{IN}}{ }^{0}$ of $4 \cdot 10^{5} \mathrm{~s}^{-1}$, a distance to the transition barrier of only $0.06 \mathrm{~nm}$, and an equilibrium force of $113 \mathrm{pN}$ where $k_{\mathrm{NI}}{ }^{0}={k_{\mathrm{IN}}}^{0}=2.8 \cdot 10^{4} \mathrm{~s}^{-1}$. This results in an equilibrium free energy difference between the native and intermediate states of $\sim 4.1 k_{\mathrm{B}} T$, in agreement with the expected energy of three hydrogen bonds. Although the absolute values of the calculated rates should be interpreted with care, the refolding rate of $4 \cdot 10^{5} \mathrm{~s}^{-1}$ is in excellent agreement with the lower limit $\left(\sim 10^{5} \mathrm{~s}^{-1}\right)$ determined directly through observation of reformed A-B $\beta$-strand pairs in high-velocity unfolding traces (Fig. S3). This suggests fast dynamic equilibrium of $\beta$-strands $A$ and $B$ at pulling forces up to $(\sim 100 \mathrm{pN})$, probably maintained by the antiparallel structure, consistent with equilibration and during pulling in SMD runs $(12,15)$. Furthermore, a recent computational small protein folding study reported a $21 \mu$ s average time to fold an antiparallel three $\beta$-strand domain (34). This suggests a novel insight in the $\beta$-sheet $A-B$ and maybe short $\beta$-folds in general: unfolding at fast and refolding at even faster rates as a feature of their structural equilibrium.

The combination of SMD with experimental FS has been important in understanding protein unfolding and mechanical stability. Our HS-FS methodology provides pulling velocities over six orders of magnitude and provides $\mu$ s time resolution achieving rates comparable to SMD simulations, and thus allowing direct comparison of experimental and simulated unfolding forces. We expect that the now accessible dynamic range of HS-FS will stimulate the development of novel theories. Our results propose detailed mechanisms of the various steps during titin 191 unfolding: At zero and moderate forces, the protein fluctuates between the native and intermediate states. Under increasing force, only the intermediate state is populated. Thus the tethered molecule reveals slow diffusion along the unfolding pathway that combined to a high energy barrier results in high mechanical stability. Direct comparison of FS and SMD simulations will likely provide new insights into other important biological processes, such as lipid membrane dynamics (35) and receptor/ligand unbinding $(7,9)$. 
Rico et al. 2013 - Experiment meets simulation: HS-FS - Science 
A

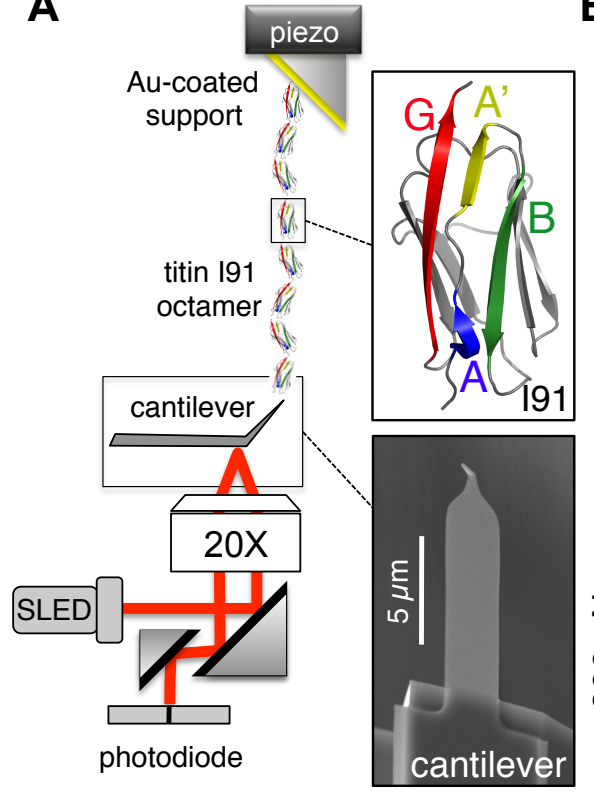

B
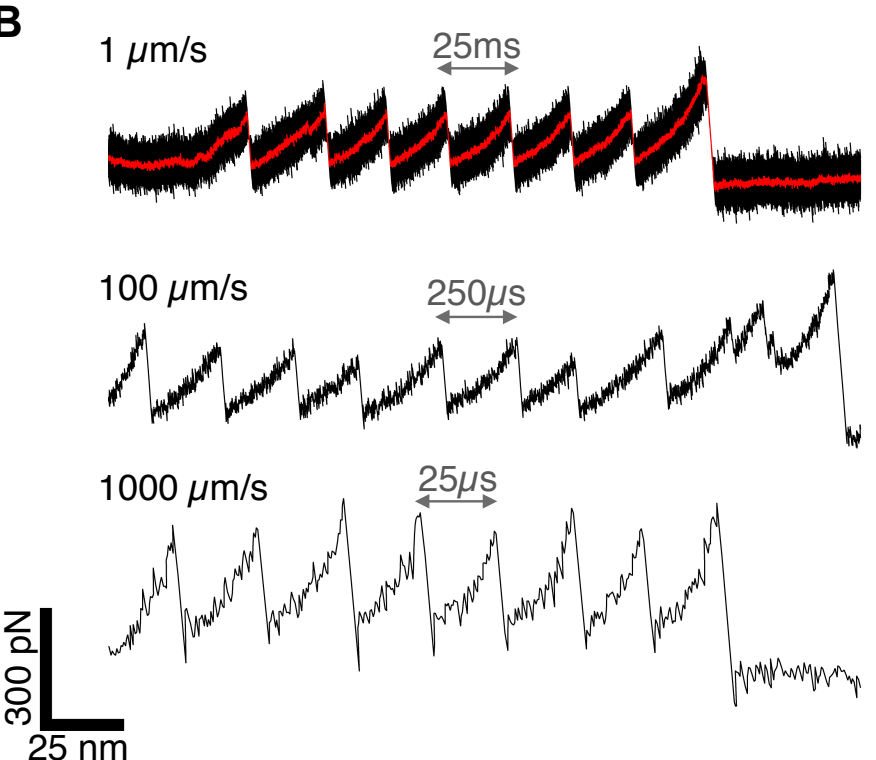

Fig. 1) High-speed force spectroscopy (HS-FS).

(A) HS-FS setup. An objective focuses the beam of the superluminescent light emitting diode and collects the light reflected by the cantilever, finally detected by a segmented photodiode. Titin 191 concatemers of 8 domains are immobilized on a tilted gold-coated surface via C-terminal cysteins. They were pulled by their $\mathrm{N}$-terminal histidine-tag with a nickel-coated tip at the end of a short cantilever. Tilting the sample surface further reduced hydrodynamic forces. Top inset shows a titin 191 domain (PDB 1TIT) with relevant $\beta$-strands colored in blue $(A)$, yellow $\left(A^{\prime}\right)$, green $(B)$ and red $(G)$. Bottom inset shows a scanning electron micrograph of a short cantilever.

(B) Force-extension curves acquired at three different retraction velocities $(1 \mu \mathrm{m} / \mathrm{s}, 100 \mu \mathrm{m} / \mathrm{s}, 1000 \mu \mathrm{m} / \mathrm{s})$. The $1 \mu \mathrm{m} / \mathrm{s}$ curve is moving average filtered (red trace, $65 \mu$ s-time window). Times to unfold single 191 domain are indicated by arrows. 

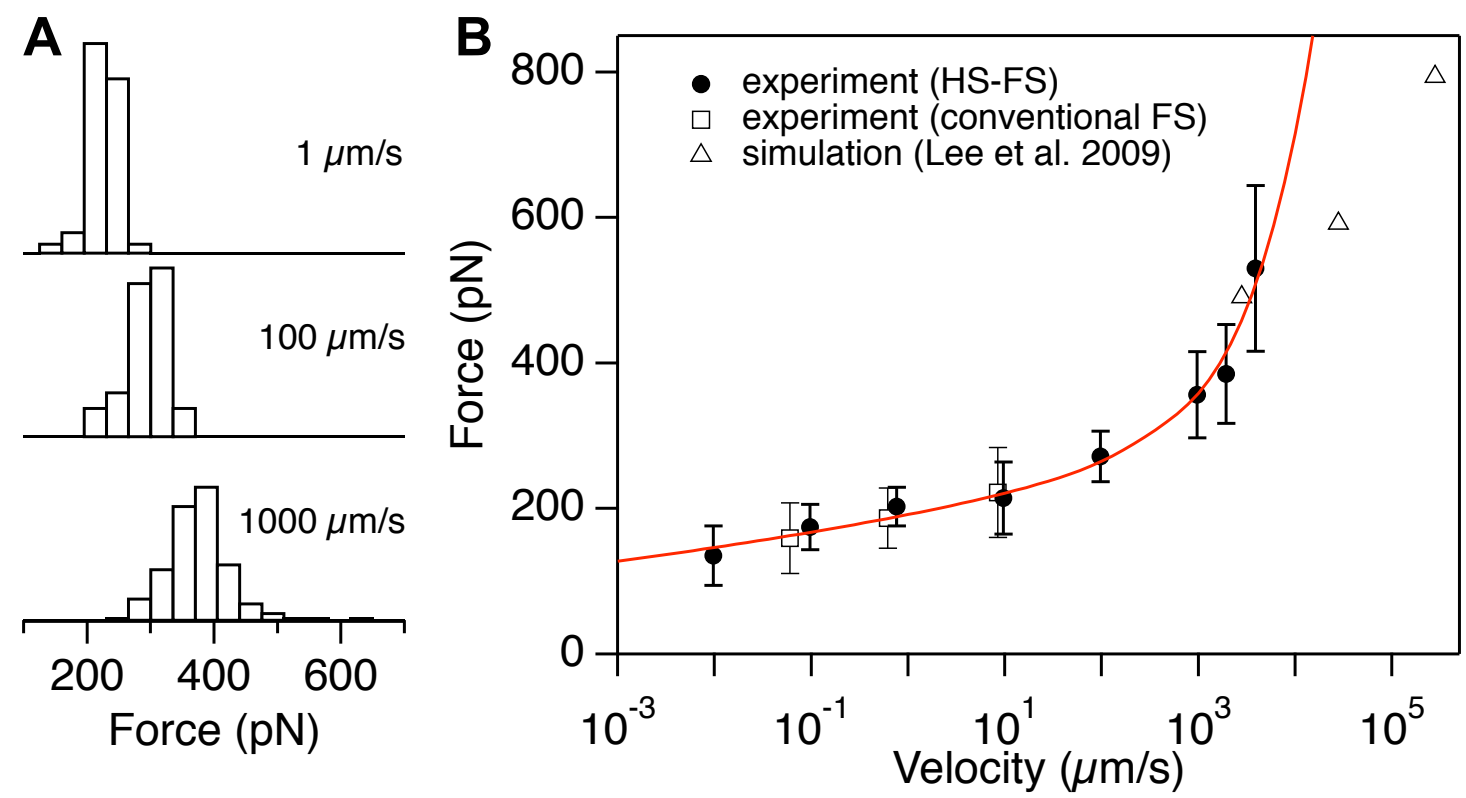

Fig. 2) High-speed dynamic force spectrum of titin 191.

(A) Unfolding force histograms of the $1 \mu \mathrm{m} / \mathrm{s}, 100 \mu \mathrm{m} / \mathrm{s}$ and $1000 \mu \mathrm{m} / \mathrm{s}$ retraction velocity experiments.

(B) Average unfolding forces versus retraction velocity obtained using HS-FS $(\bullet$, error bars denote standard deviations), conventional FS ( $\square$ ) and steered molecular dynamics simulations ( $\Delta$, data from Lee et al. (15)). Solid red line is the fit to the entire dynamic range of HS-FS with the full microscopic model (26) with fitting parameters $( \pm \mathrm{SD})$ of $x_{\beta}=0.89 \pm 0.05 \mathrm{~nm}, D=3925 \pm 183 \mathrm{~nm}^{2} / \mathrm{s}$ and $k_{\mathrm{m}}=376 \pm 28 \mathrm{pN} / \mathrm{nm}$. 

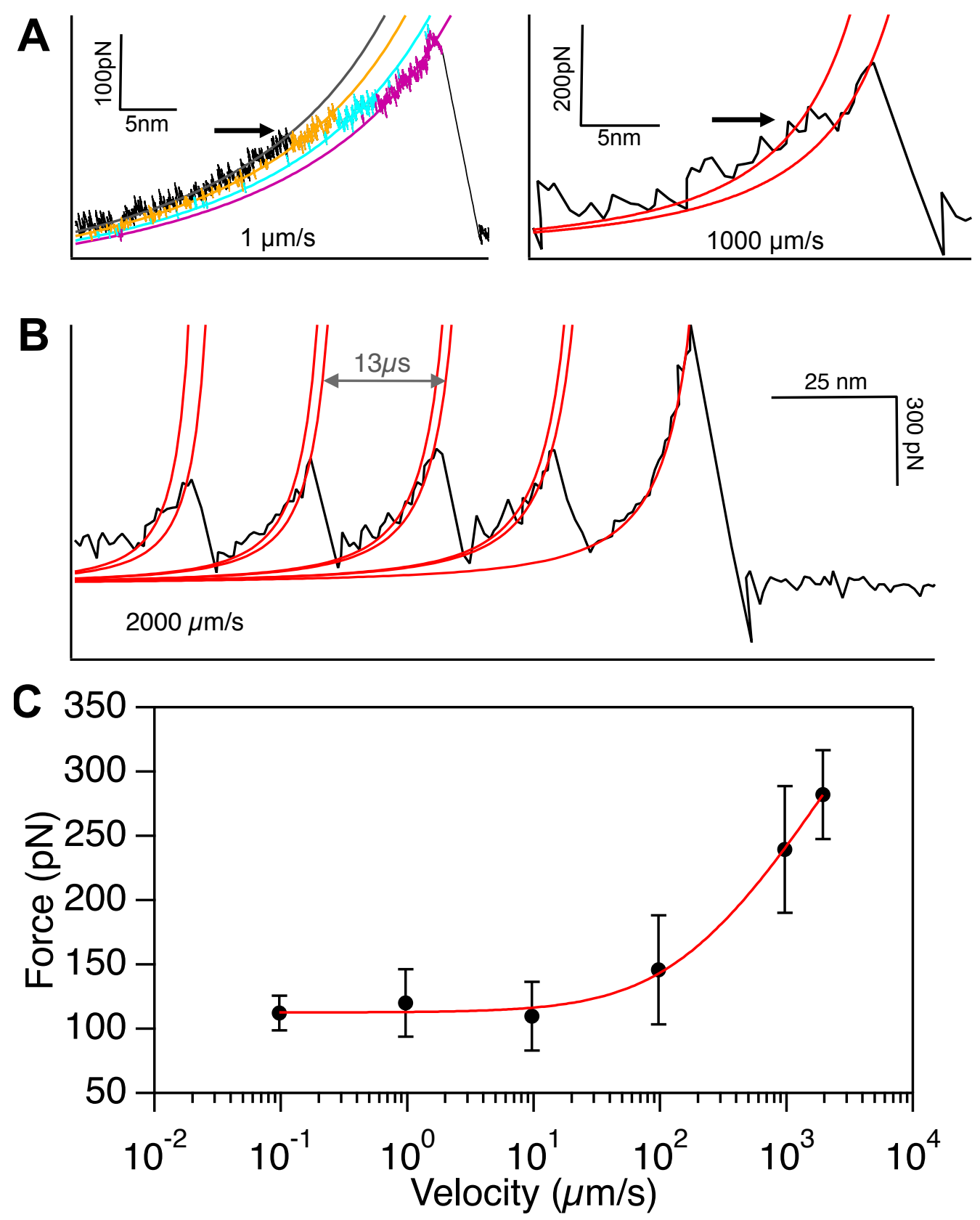

Fig. 3) High-speed force spectroscopy of unfolding intermediate.

(A) (Left) Force-extension trace at $1 \mu \mathrm{m} / \mathrm{s}$ (moving average filtered with $65 \mu \mathrm{s}$ time window) showing the intermediate unfolding state "hump" (arrow) separating antiparallel $\beta$-strands $A$ and $B$. Cantilever fluctuations above the noise level of the trace are interpreted as hopping between intermediate states of the remaining folded domains (see Fig. S5). Colored lines are worm-like chain (WLC) model fits. (Right) Force-extension trace (black line) at $1000 \mu \mathrm{m} / \mathrm{s}$ showing the intermediate unfolding state "hump" (arrow). Red lines are the best 
fits of the WLC model to the hump and complete unfolding peaks. The difference between the contour lengths is consistent with a separation of $n$ times $0.7 \mathrm{~nm}$ of each A-B $\beta$-strand pair of the remaining folded domains (11).

(B) Force-extension curve at $2000 \mu \mathrm{m} / \mathrm{s}$ showing unfolding of four 191 domains. Red lines are WLC fits to the "hump" and complete unfolding peaks. The contour length distance difference between the "hump" and the complete unfolding decreases with the decreasing number of remaining folded domains. (C) Dynamic force spectrum of the intermediate unfolding state. Solid red line is the best fit of the model developed by Friddle et al. (33) to the experimental data with fitting parameters $( \pm \mathrm{SD}) x_{t}=0.060 \pm 0.004 \mathrm{~nm}, f_{\mathrm{eq}}=113 \pm 1 \mathrm{pN}$ and $k_{\mathrm{NI}}=6959\left(\begin{array}{c}+1398 \\ -990\end{array}\right) \mathrm{s}^{-1}$.

\section{References}

1. D. S. Herman et al., New Engl. J. Med. 366, 619 (2012).

2. $\quad$ K. Maruyama et al., J. Biochem. (Tokyo). 82, 317 (1977).

3. M. s. S. Z. Kellermayer, S. B. Smith, H. L. Granzier, C. Bustamante, Science 276, 1112 (1997).

4. L. Tskhovrebova, J. Trinick, J. A. Sleep, R. M. Simmons, Nature 387, 308 (1997).

5. M. Rief, M. Gautel, F. Oesterhelt, J. M. Fernandez, H. E. Gaub, Science 276, 1109 (1997).

6. H. Grubmuller, B. Heymann, P.f Tavan, Science 271, 997 (1996).

7. S. Izrailev, S. Stepaniants, M. Balsera, Y. Oono, K. Schulten, Biophys. J. 72, 1568 (1997).

8. H. Lu, B. Isralewitz, A. Krammer, V. Vogel, K. Schulten, Biophys. J. 75, 662 (1998).

9. $\quad$ E. L. Florin, V. T. Moy, H. E. Gaub, Science 264, 415 (1994).

10. G. U. Lee, L. A. Chrisey, R. J. Colton, Science 266, 771 (1994).

11. P. E. Marszalek et al., Nature 402, 100 (1999).

12. H. Lu, K. Schulten, Biophys. J. 79, 51 (2000).

13. S. B. Fowler et al., J. Mol. Biol. 322, 841 (2002).

14. R. B. Best et al., J. Mol. Biol. 330, 867 (2003).

15. E. H. Lee, J. Hsin, M. Sotomayor, G. Comellas, K. Schulten, Structure 17, 1295 (2009).

16. T. Ando et al., Proceedings of the National Academy of Sciences 98, 12468 (2001).

17. T. Uchihashi, R. Iino, T. Ando, H. Noji, Science 333, 755 (2011).

18. N. Kodera, D. Yamamoto, R. Ishikawa, T. Ando, Nature 468, 72 (2010).

19. I. Casuso et al., Nat Nano 7, 525 (2012).

20. T. Ando, T. Uchihashi, T. Fukuma, Prog. Surf. Sci. 83, 337 (2008).

21. M. B. Viani et al., J. Appl. Phys. 86, 2258 (1999). 
22. R. B. Best et al., Anal. Chim. Acta 479, 87 (2003).

23. M. Carrion-Vazquez et al., Proceedings of the National Academy of Sciences 96, 3694 (1999).

24. R. Merkel, P. Nassoy, A. Leung, K. Ritchie, E. Evans, Nature 397, 50 (1999).

25. X. H. Zhang, E. Wojcikiewicz, V. T. Moy, Biophys. J. 83, 2270 (2002).

26. G. Hummer, A. Szabo, Biophys. J. 85, 5 (2003).

27. Z. T. Yew, M. Schlierf, M. Rief, E. Paci, Physical Review E 81, 031923 (2009).

28. O. K. Dudko, G. Hummer, A. Szabo, Phys. Rev. Lett. 96, (2006).

29. L. J. Lapidus, W. A. Eaton, J. Hofrichter, Proceedings of the National Academy of Sciences 97, 7220 (2000).

30. R. Berkovich et al., Proceedings of the National Academy of Sciences 109, 14416 (2012).

31. H. Lannon, J. S. Haghpanah, J. K. Montclare, E. Vanden-Eijnden, J. Brujic, Phys. Rev. Lett. 110, 128301 (2013).

32. H. Li et al., Nature 418, 998 (2002).

33. R. W. Friddle, A. Noy, J. J. De Yoreo, Proceedings of the National Academy of Sciences 109, 13573 (2012).

34. K. Lindorff-Larsen, S. Piana, R. O. Dror, D. E. Shaw, Science 334, 517 (2011).

35. L. Redondo-Morata, M. I. Giannotti, F. Sanz, Langmuir 28, 6403 (2012).

36. H. J. Butt, M. Jaschke, Nanotechnology 6, 1 (1995).

37. J. L. Hutter, J. Bechhoefer, Rev. Sci. Instrum. 64, 1868 (1993).

38. J. M. Nunes et al., Angew. Chem. Int. Ed. 49, 3528 (2010).

39. P. M. Williams et al., Nature 422, 446 (2003).

Acknowledgments: The authors thank Alfonso Sastre and Jordi Otera for helpful discussions. This study was supported by a European Research Council (ERC) Starting Grant (\#310080), and an Agence National de la Recherche (ANR) grant (\# ANR-12-BS10-009-01). Laura Gonzalez was recipient of a travel grants (FPI grant BES-2010-031186 from the Spanish Ministerio de Economía y Competitividad) and (STSM COST Action TD1002 - 10006).

\section{Supplementary Materials:}

Materials and Methods

SupplementaryText

Figs. S1 to S6 